\title{
Estudo da Farmacoterapia da Dor em Pacientes Pós-cirúrgicos do Hospital de Clínicas da Universidade Federal do Paraná
}

\author{
Pharmacotherapy Study of Pain In Post-surgical Patients \\ at Hospital de Clínicas of the Federal University of Paraná
}

Vladimir ANTONINI ${ }^{*}$, Elizabeth TAMBARA ${ }^{1}$, Marcelo Martins BAVIERA², Carolina Rizzoni SILVEIRA²,

Eduardo Dias de SOUZA ${ }^{4}$

'Programa de Pós-Graduação em Clínica Cirúrgica, Setor de Ciências da Saúde; Universidade Federal do Paraná, Curitiba, Paraná. ${ }^{2}$ Hospital de Caridade da Irmandade da Santa Casa de Misericórdia de Curitiba, Paraná. ${ }^{3}$ Pontificia Universidade Católica do Paraná, Curitiba, Paraná. E-mail: antonini@ufpr.br

\section{ABSTRACT}

Post-operatory pain is an acute pain difficult to effectively control. The object of this study was to assess, as compared to what is found in international literature, the incidence of post-operatory pain in patients submitted to digestive tract surgery, managed with standard analgesic and anti-inflammatory drugs at Hospital de Clínicas, the university hospital of the Federal University of Paraná, UFPR. One hundred patients of both genders, age group between 19 and 65 years, were followed from the Immediate Post- Anesthetic Recovery Unit until discharge, or up to 72 hours after surgery, through a double blind study consisting of oral questions about pain feeling and intensity. We compared the pain rate in patients who were given dypirone and dypirone plus ketoprofen, or dypirone plus tramadol, or dypirone plus ketoprofen and tramadol at given times. Of the 91 patients examined at the Immediate Post-Anesthetic Recovery Unit, 33 reported pain. Among the 93 patients followed since their return to the admission unit until the first drug administration on the following day, 30 reported pain. On day one post-op, 93 patients were followed of which 24 reported pain. On day two, of 37 patients, 11 still had pain. There was no statistically significant difference among patients who were given dypirone alone or associated with other drugs. This study showed that the incidence of acute continuous post-op pain in patients at Hospital de Clínicas of the UFPR managed with standard analgesic and anti- inflammatory drugs was thirty percent, the minimal margin shown in international literature.

KEY WORDS: Post-operatory pain, Analgesia, Analgesic drugs, Anesthesia, Anesthetics, Acute pain.

\section{RESUMO}

A dor pós-operatória é uma dor aguda de difícil controle efetivo. O objetivo deste estudo foi avaliar, comparativamente com a literatura internacional, a incidência de dor pós-operatória nos pacientes submetidos a operações do aparelho digestivo, tratados com os analgésicos e anti-inflamatórios padronizados no Hospital de Clínicas da UFPR. Cem pacientes de ambos os sexos, com idade entre 19 e 65 anos, foram acompanhados desde a Unidade de Recuperação Pós- Anestésica Imediata até a alta hospitalar, ou até 72 horas após a cirurgia, através de ensaio duplamente encoberto com perguntas verbais sobre a presença e a intensidade da dor. Foi comparado o índice de dor dos pacientes que receberam dipirona e dipirona associada a cetoprofeno ou dipirona associada a tramadol ou dipirona associada a cetoprofeno e tramadol nos momentos determinados. De 91 pacientes analisados na Unidade de Recuperação Pós-anestésica Imediata, 33 reportaram dor. Entre os 93 pacientes observados desde o retorno à unidade de internação até a primeira administração de medicação do dia seguinte, 30 apresen- 
taram dor. No primeiro dia do período pós- operatório, 93 pacientes foram acompanhados, sendo encontrados 24 pacientes com dor. No segundo dia, de 37 pacientes internados, 11 continuavam com dor. Não houve diferença estatisticamente significativa entre os pacientes que receberam dipirona isolada ou associada a outros medicamentos. O presente estudo demonstrou que a incidência de dor aguda contínua pós-operatória nos pacientes do Hospital de Clínicas da Universidade Federal do Paraná tratados com os analgésicos e anti- inflamatórios padronizados foi de trinta por cento, margem mínima indicada na literatura internacional.

PALAVRAS-CHAVE: Dor Pós-operatória, Analgesia, Analgésicos, Anestesia, Anestésicos, Dor Aguda.

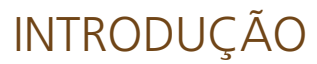

Desde que o homem tomou consciência de seu ambiente, de sua capacidade e, sobretudo, de suas limitações, a dor é um de seus tormentos; uma sensação que aterroriza tanto quanto, ou até mais, que a fome, o frio ou mesmo a morte. Em qualquer procedimento invasivo feito em pacientes, é imprescindível garantir a ausência ou diminuição substancial da dor e dos desconfortos maiores que as limitações de movimento ou restrições de dieta possam acarretar (1).

Em procedimentos cirúrgicos, são dois os tipos de dores encontrados: aguda e crônica.

A dor aguda está relacionada a afecções traumáticas, infecciosas ou inflamatórias, é caracterizada por apresentar delimitação têmporo-espacial precisa, respostas neurovegetativas associadas (elevação da pressão arterial, taquicardia, taquipnéia, entre outros), causar frequentemente ansiedade e agitação psicomotora, ter a função biológica de alertar o organismo sobre a agressão, com expectativa de desaparecimento após a cura da lesão (2).

A dor aguda pode ser contínua ou intermitente, produzida por movimentos. Espera-se que o paciente sinta apenas a dor intermitente, sendo esta fundamental para alertar o indivíduo que ele não pode se mover ou exagerar nos movimentos. É uma dor conservativa no sentido estrito do termo porque sua presença tem como função fisiológica, prevenir danos ao organismo do paciente (3).

A dor aguda contínua pós-operatória pode persistir por 24 ou até 48 horas (4).

Dor crônica é aquela que persiste após o tempo razoável para a cura de uma lesão ou que está associada a processos crônicos, que causam dor contínua ou recorrente. Não tem mais a função biológica de alerta, geralmente não há respostas neurovegetativas associadas ao sintoma, é mal delimitada no tempo e no espaço e ansiedade e depressão são respostas emocionais frequentemente associadas ao quadro (2).

A maioria dos hospitais trata a dor aguda pós- procedimento cirúrgico com fármacos analgésicos e anti- inflamatórios e no Hospital de Clínicas da Universidade Federal do Paraná não é diferente. Os medicamentos são padronizados pela Comissão de Farmácia e Terapêutica, sendo prescritos pelos médicos no Sistema de Informática Hospitalar (SIH), dispensados pela Equipe de Dispensação á Internados da Unidade de Farmácia Hospitalar e administrados pelas Equipes de Enfermagem das Unidades de Internação Cirúrgicas aos seus pacientes conforme dose, posologia e recomendações constantes nas prescrições médicas (3).

No curso da história da humanidade a dor já foi usada com todo tipo de finalidade, desde mecanismo de escape de responsabilidades ou imposições, até como tratamento de loucura, bruxaria, feitiçaria e satanismo. O romano Celsus (Aulus Cornellius Celsus, $25 \mathrm{aC}-45$ $\mathrm{dC}$ ), autor do livro De Re Medicina, primeira obra reconhecida historicamente como um manual de psiquiatria, prescrevia tratamentos rudes e à base de dor para curar a loucura dos doentes do seu tempo. Os pacientes eram submetidos a torturas físicas com equipamentos e dispositivos que produziam altos níveis de dor e os que não saíam do surto, eram queimados em fogueiras e, conforme Celsus dizia, "o doente morreu curado" $(5,6)$.

Passaram-se dos milênios desde a publicação das idéias de Celsius e durante todo este tempo, muita coisa mudou. Praticamente todos os mitos foram desvendados e abandonados, mas o tratamento da dor continua sendo um problema para os profissionais de saúde devido às diferentes facetas que o fenômeno doloroso assume $(5,6)$.

Centenas de compostos com ação analgésica foram descobertos ao longo dos séculos. Inúmeras teorias que tentavam explicar o mecanismo da dor foram propostas, mas a dor no pós-operatório imediato mantém-se como uma entidade a ser vencida $(5,6)$, tanto que ainda hoje, os pesquisadores são unânimes em apontar uma prevalência entre $30 \%$ e $60 \%$ de dor aguda contínua no pós-operatório imediato a nível mundial $(14,15,16,17,18)$.

Dentre os inúmeros fármacos desenvolvidos estão a dipirona, o tramadol e o cetoprofeno - os mais utilizados pelos hospitais brasileiros para este tipo de condição. 
A dipirona foi patenteada em 1911, sob no 254 , pelo Laboratório Hoerst da Alemanha que a batizou com o nome comercial de Metamizol, enquanto o tramadol e o cetoprofeno são mais recentes $(5,7)$.

Dipirona é o principal nome genérico de ácido [(2,3- dihidro-1,5-dimetil-3-oxo-2-fenil-1H-pirazol-4il)metilamino] metanossulfônico, também chamado de 1- fenil-2,3-dimetil-5-pirazolona-4-metilamino- metanossulfonato de sódio (ou de magnésio), e ainda denominado de metamizol, um segundo nome genérico muito encontrado na literatura farmacológica norte- americana e européia (7).

Uma revisão Cochrane publicada primeiramente em 2001 e revisada em 2010 analisou a eficácia e a frequência de efeitos adversos de uma dose única de dipirona sobre dor pós-operatória. Quinze estudos testaram principalmente $500 \mathrm{mg}$ de dipirona via oral (173 participantes), 2,5g de dipirona via intravenosa (101 participantes), 2,5g de dipirona via intramuscular (99 participantes); menos de 60 participantes receberam outras doses (8).

Todos os estudos utilizaram a comparação com outro fármaco (ibuprofeno, paracetamol, ácido acetilsalicílico, flurbiprofeno, cetorolaco, cetoprofeno, tramadol, entre outros) e oito estudos utilizaram placebo, $70 \%$ dos participantes obtiveram redução em pelo menos $50 \%$ da dor durante 4 a 6 horas após o uso de $500 \mathrm{mg}$ via oral, comparados a $30 \%$ com placebo (cinco estudos, 288 participantes) (8).

Os participantes que receberam dipirona também necessitaram de menos medicação de resgate $(7 \%) \mathrm{em}$ relação àqueles que receberam placebo $(34 \%$, quatro estudos, 248 participantes). Não houve diferença entre os participantes que relataram alívio de no mínimo $50 \%$ da dor com $2,5 \mathrm{~g}$ de dipirona via intravenosa ou com $100 \mathrm{mg}$ de tramadol via intravenosa $(70 \%$ e $65 \%$, respectivamente, dois estudos, 200 participantes). Não houve relatos de efeitos adversos sérios (8).

Assim, baseado nessas poucas informações disponíveis, acredita-se que a dipirona $500 \mathrm{mg}$ traga alívio na dor pós-operatória em $70 \%$ dos pacientes (8).

Em dois estudos (113 participantes), a taxa de resposta média à administração oral de $1 \mathrm{~g}$ de dipirona foi de $69 \%$ em comparação com $20 \%$ após placebo (8).

Em outro estudo (70 participantes), a taxa de resposta com administração intramuscular de $2 \mathrm{~g}$ de dipirona foi de $74 \%$, versus $46 \%$ com placebo. Percebeu-se, dessa maneira, que doses de $500 \mathrm{mg}$ a $2 \mathrm{~g}$ determinaram resposta analgésica similar (9).

O tramadol, que é constituído de uma mistura racêmica e possui ação analgésica tanto através de receptores opioides quanto por aumento nos níveis de serotonina e noradrenalina, ainda não teve divulgada uma revisão de literatura com análise da sua ação em dores pós-operatórias. Apesar de alguns autores declararem que o tramadol é eficaz no tratamento de dores pós-operatórias agudas, moderadas e intensas, poucos estudos demonstraram a sua efetividade como fármaco isolado, incluindo a relação desta ação com a dose utilizada (10).

Calcula-se que, com uma dose de $86 \mathrm{mg}$ (bastante próxima da dose usual de $100 \mathrm{mg}$ ), consegue-se alívio na dor de $50 \%$ dos pacientes, subentendendo-se, então, que sua efetividade seja próxima de $50 \%$. Com o aumento da dose, no entanto, é possível aumentar a sua efetividade. Para obter-se o alívio da dor pós-operatória em no mínimo $80 \%$ dos pacientes, um estudo (dois grupos com 24 pacientes cada) demonstrou que deve ser usada uma dose de $260 \mathrm{mg}$ IV, o que é muito acima da dose recomendada pelos fabricantes. Assim, os autores desse estudo não recomendam a utilização do tramadol como fármaco isolado nesse tipo de dor (10).

$O$ cetoprofeno também é uma mistura racêmica, existindo um isômero levógiro e um dextrógiro. $\mathrm{O}$ isômero dextrógiro é o elemento ativo e por isso também sendo chamado de dexcetoprofeno.

Uma revisão Cochrane publicada em 2008 encontrou trinta e cinco ensaios clínicos com 6.380 pacientes com dor aguda ou crônica comparando o cetoprofeno, em doses variadas, com placebo, demonstrando superioridade do cetoprofeno em relação ao placebo no alívio de $50 \%$ da dor (11).

Um estudo de eficácia comparativa entre cetoprofeno e dipirona, com 120 pacientes, apontou superioridade analgésica do cetoprofeno na dose $25 \mathrm{mg}$ na extração do terceiro molar $(12,13)$.

Dessa forma, o objetivo deste estudo foi avaliar, comparativamente com a literatura internacional, a incidência de dor aguda contínua pós-operatória nos pacientes das Unidades de Internação de Cirurgia do Aparelho Digestivo e Cirurgia Geral do Hospital de Clínicas da Universidade Federal do Paraná e a eficácia do esquema analgésico e anti-inflamatório padronizado e utilizado no tratamento dos clientes.

\section{MATERIAIS E MÉTODO}

Este estudo foi realizado nas Unidades de Internação Cirurgia do Aparelho Digestivo (5 - CAD) e Cirurgia Geral (7 - CG) do Hospital de Clínicas da Universidade Federal do Paraná, no período de 10 de janeiro de 2011 à 20 de fevereiro de 2011.

O estudo foi aprovado pelo Comitê de Ética em Pesquisa em Seres Humanos do Hospital de Clínicas da Universidade Federal do Paraná (CEP-HC-UFPR), registro CEP: 2385.279/2010-11, CAE: 0310.0.208.00010, em 30 de novembro de 2010. 


\section{Amostra}

Cento e quatro pacientes assinaram o Termo de Consentimento Livre e Esclarecido (TCLE), condição sine qua non para inclusão no estudo.

Foram incluídos pacientes de ambos os sexos, com idade entre 19 e 65 anos, submetidos a operações do aparelho digestivo.

Foram excluídos pacientes com histórico de cirurgias anteriores ou patologias com experimentação de dor intensa no passado e pacientes com doenças crônicas do fígado (cirrose alcoólica, portadores dos vírus $\mathrm{B}$ e C de hepatite).

\section{Método}

Este estudo adotou o ensaio duplamente encoberto como metodologia, ou seja, tanto os pacientes, quanto o pesquisador, não sabiam qual analgésico era ministrado, assim como os dados por entrevista, foram coletados sem verificação ou comparação imediata com as informações dos prontuários, estas levantadas ad posteriori e confrontadas com os resultados obtidos.

A entrevista consistiu de quatro perguntas simples, de sim ou não, feitas verbalmente, de acordo com um roteiro, e de uma avaliação sobre a intensidade da dor em escala numérica de 0 a 10 .

Foi comparado o índice de dor da literatura com $\mathrm{o}$ apresentado pelos pacientes que receberam dipirona $\mathrm{e}$ dipirona associada a cetoprofeno ou dipirona associada a tramadol ou dipirona associada a cetoprofeno e tramadol nos momentos determinados.

Foram cinco os momentos de avaliação:

REPAI (Recuperação Pós-Anestésica Imediata) período após o término do procedimento cirúrgico, entre a chegada à sala da Recuperação Pós-anestésica Imediata e a saída para a Unidade de Internação, cuja permanência média foi de duas horas e trinta minutos.

0 POI (Zero Pós-operatório Imediato) - é o intervalo de tempo compreendido entre a chegada do paciente à Unidade de Internação e o primeiro horário de administração de medicamentos de rotina, às duas horas da manhã.

$1^{\circ}$ POI (Primeiro Pós-operatório) - período de 24 horas à partir das duas horas do dia seguinte ao procedimento cirúrgico.

$2^{\circ}$ POI (Segundo Pós-operatório) - período de 24 horas à partir das duas horas do $1^{\circ}$ POI.

$3^{\circ}$ POI (Terceiro Pós-operatório) - período de 24 horas à partir das duas horas do $2^{\circ}$ POI.

As respostas dadas pelos pacientes nas entrevistas foram comparadas com as anotações da enfermagem nos prontuários dos pacientes.

\section{Análise Estatística}

Para fins de análise estatística, fixou-se uma taxa de trinta por cento $(30 \%)$ de pacientes com dor, tendo por base dois fatores: a eficácia analgésica da dipirona, estimada em $70 \%$ (8) e a porcentagem de pacientes que relatam presença de dor segundo a literatura internacional, entre trinta e cinquenta por cento $(30-50 \%)$ $(14,15,16,17,18)$.

O teste não-paramétrico aplicado foi o Qui-quadrado (X2) para cada caso em comparação entre dipirona e a associação de dipirona com outro fármaco para os pacientes com dor, aplicando-se a correção de Yates quando necessário. Este teste também foi utilizado na comparação entre o número de pacientes com dor durante as cinco etapas da pesquisa (REPAI, OPOI, $1^{\circ} \mathrm{POI}, 2^{\circ} \mathrm{POI}$ e $\left.3^{\circ} \mathrm{POI}\right)$. Os dados levantados nas entrevistas foram processados no software livre BioStat 5.

\section{Materiais}

Todos os fármacos estudados foram adquiridos pela Unidade de Abastecimento do Hospital de Clínicas da UFPR em conformidade com a legislação em vigor, não havendo conflito de interesses nos resultados obtidos.

Fizeram parte dos materiais deste trabalho as prescrições médicas digitadas no Sistema de Informática Hospitalar (SIH), as prescrições médicas impressas, as prescrições de enfermagem, os quadros de anotações de enfermagem, as folhas de evolução de enfermagem, as folhas de dispensação e as de devolução de medicamentos.

\section{RESULTADOS}

REPAI: Dos 104 pacientes selecionados para participarem deste trabalho, quatro foram excluídos devido ao uso de analgesia opioide por cateter epidural.

Foram acompanhados 91 pacientes na REPAI, porque nove anotações sobre os medicamentos utilizados nesta fase estavam incompletas.

Trinta e três pacientes relataram dor na Recuperação Pós-anestésica Imediata, sendo 10 com dor aguda fraca, 12 com dor moderada e 11 com dor intensa. Eram esperados 27,4 pacientes com dor e foram observados 33 pacientes, para um $p<0,20$, não houve significância na variação da amostra.

A utilização venosa da dipirona isolada comparada com a associada ao cetoprofeno, na REPAI, não apresentou diferença estatisticamente significativa (praticamente nula), obtendo um $p>0,95$ (Tabela 1 ). 
Tabela 1: Medicação (intravenosa) versus dor na fase de recuperação pós-anestésica imediata (REPAI)

\begin{tabular}{|l|c|c|c|}
\hline \multirow{2}{*}{ Medicação } & \multicolumn{3}{c|}{ Pacientes } \\
\cline { 2 - 4 } & Com dor & Sem dor & Total \\
\hline dipirona & 5 & 11 & 16 \\
\hline dipirona + cetoprofeno & 21 & 46 & 67 \\
\hline
\end{tabular}
$p>0,95$

0 POI: Sete pacientes não tinham documentação preenchida adequadamente em seus prontuários, resultando em 93 pacientes acompanhados nesta fase. Dentre os 93 pacientes observados, eram esperados 27,9 pacientes com dor e foram observados 30 , resultando em um $p>0,20$, não havendo diferença estatística entre o esperado e o observado, ficando dentro dos limites da literatura.

No 0 POI, foram analisados apenas 60 pacientes porque 30 fichas estavam com anotação incompleta. Não houve diferença estatística entre os pacientes que receberam dipirona isolada e os que foram tratados com a associada com tramadol ou associada com tramadol e cetoprofeno $(\mathrm{p}>0,50)$ (Tabela 2$)$.

Tabela 2: Medicação (intravenosa) versus dor na fase Zero pós-operatório imediato (0 POI)

\begin{tabular}{|l|c|c|c|}
\multirow{2}{*}{\multicolumn{1}{c|}{ Medicação }} & \multicolumn{3}{c|}{ Pacientes } \\
\cline { 2 - 4 } & Com dor & Sem dor & Total \\
\hline Dipirona & 5 & 12 & 17 \\
\hline Dipirona + Tramadol & 6 & 16 & 22 \\
\hline $\begin{array}{l}\text { Dipirona + Cetoprofeno + Tramadol } \\
\text { p }>0,20\end{array}$ & 9 & 12 & 21 \\
\hline
\end{tabular}

$1^{\circ}$ POI: Dos 93 pacientes, eram esperados 28 pacientes com dor e foram observados 24 . Não houve diferença significativa entre o número esperado e o encontrado ( $>00,20)$.

Não houve diferença estatística entre os pacientes que receberam dipirona isolada e os que foram tratados com dipirona associada com tramadol, dipirona associada com cetoprofeno ou associada a ambos, tramadol e cetoprofeno (Tabela 3 ).

Tabela 3: Medicação (intravenosa) versus dor na fase Primeiro pós-operatório $\left(1^{\circ} \mathrm{POI}\right)$

\begin{tabular}{|l|c|c|c|}
\multirow{2}{*}{ Medicação } & \multicolumn{4}{c|}{ Pacientes } \\
\cline { 2 - 4 } & Com dor & Sem dor & Total \\
\hline Dipirona & 2 & 13 & 15 \\
\hline Dipirona + Cetoprofeno & 4 & 7 & 11 \\
\hline Dipirona + Tramadol & 7 & 23 & 30 \\
\hline Dipirona + Cetoprofeno + Tramadol & 4 & 14 & 18 \\
\hline p $>0,20$ & & & \\
\hline
\end{tabular}

$2^{\circ}$ POI: Entre os 37 pacientes avaliados, eram esperados 11,1 com dor e foram observados 11. Para um $\mathrm{p}>0,95$, não houve diferença entre o esperado e o observado. Da mesma forma, não existiu diferença entre a utilização de dipirona isolada ou a associada a outro fármaco (Tabela 4).

Tabela 4: Medicação (intravenosa) versus dor na fase Segundo pós-operatório ( $\left.2^{\circ} \mathrm{POI}\right)$

\begin{tabular}{|l|c|c|c|}
\multirow{2}{*}{\multicolumn{1}{c|}{ Medicação }} & \multicolumn{4}{c|}{ Pacientes } \\
\cline { 2 - 4 } & Com dor & Sem dor & Total \\
\hline Dipirona & 3 & 5 & 8 \\
\hline Dipirona + Cetoprofeno & 1 & 5 & 6 \\
\hline Dipirona + Tramadol & 2 & 7 & 9 \\
\hline Dipirona + Cetoprofeno + Tramadol & 1 & 3 & 4 \\
\hline p $>0,20$ & & & \\
\hline
\end{tabular}

$3^{\circ} \mathrm{POI}$ : Foram avaliados 26 pacientes e era esperado que nenhum deles apresentasse dor, mas 8 pacientes relataram apresentar dor nessa fase. Não houve comparação estatística entre os fármacos administrados devido à ausência de poder estatístico da amostra.

Comparando-se o número de pacientes com dor e sem dor em todas as etapas, não existiu diferença estatisticamente significativa (Tabela 5).

\section{DISCUSSÃO}

Com a evolução dos conhecimentos sobre analgesia, a dor pós-operatória tem recebido muita atenção, devido às suas consequências na recuperação do paciente. Um tipo extremamente comum de queixa de dor aguda em pacientes cirúrgicos, resultante de respostas autonômicas, psicológicas e comportamentais ao trauma cirúrgico, é causa de experiência sensitiva e emocional, desagradável e indesejada $(2,17)$. Estudos mostram que sua expressão somática e psíquica pode ser associada, direta ou indiretamente, ao aumento da morbimortalidade e ao tempo de hospitalização com repercussões sociais e econômicas e sua persistência tende a ampliar a ocorrência de eventos adversos, gerando desfechos clínicos indesejados (19).

Tabela 5: Pacientes que apresentaram dor nas diversas fases pós-operatórias

\begin{tabular}{|l|c|c|}
\hline \multirow{2}{*}{\multicolumn{1}{c|}{ Fases }} & \multicolumn{2}{c|}{ Pacientes } \\
\cline { 2 - 3 } & Com dor & Sem dor \\
\hline REPAI & 33 & 58 \\
\hline 0 POI & 28 & 62 \\
\hline $1^{\circ}$ POI & 22 & 71 \\
\hline $2^{\circ}$ POI & 11 & 25 \\
\hline $3^{\circ}$ POI & 8 & 18 \\
\hline
\end{tabular}

$p>0,5$ 
O grande interesse do controle efetivo da dor no paciente cirúrgico, baseado na terapia farmacológica visa minimizar o desconforto, prevenir os efeitos deletérios e facilitar o processo de recuperação (19). Atingir esses objetivos depende do modo como a terapia analgésica é conduzida (20). Apesar do grande desenvolvimento no campo do conhecimento sobre a fisiopatologia da dor e sobre a farmacocinética e a farmacodinâmica dos medicamentos utilizados no seu tratamento, ainda existe dificuldade no controle efetivo desse sintoma, exemplo disso, são as inúmeras publicações comprovando que o fenômeno doloroso não é adequadamente controlado. A controvérsia entre os pesquisadores acerca do índice real de dor pós-operatório é frequente nos estudos conduzidos atualmente, há, no entanto, um consenso em torno de trinta a sessenta por cento, uma faixa um tanto ampla, mas aceita devido à diferença dos métodos analgésicos empregados em cada hospital (14-18).

No Hospital de Clínicas da UFPR os fármacos mais utilizados no tratamento da dor pós-operatória em aparelho digestivo são a dipirona, o cetoprofeno e o tramadol, classificados nesta ordem conforme o número de prescrições médicas e quantidade de unidades posológicas dispensadas e administradas.

O foco desta pesquisa foi a análise do índice de dor pós-operatório em um hospital universitário, em contraponto ao menor índice encontrado na literatura. Pacientes com potencial de desenvolvimento de dor por indução ou sugestão, bem como aqueles com histórico de cirurgias anteriores ou patologias com experimentação de dor intensa no passado foram excluídos do presente trabalho. Históricos de patologias e características de cada paciente foram pesquisados em entrevista preliminar, antes do convite para participar do estudo, evitando, com isso, o viés de memória. Foram excluídos, ainda, pacientes com doenças hepáticas crônicas (cirrose alcoólica, portadores dos vírus $\mathrm{B}$ e $\mathrm{C}$ de hepatite) considerando que nestas hepatopatias há diminuição da metabolização da dipirona em seus produtos ativos (4- MAA, 4-AA), o que reduz a biodisponibilidade do analgésico (21), particularidade que aumentaria o número de pacientes com dor e, posteriormente, acarretaria erro de aferição.

A coleta de dados dos pacientes foi passiva, através de estudo duplamente encoberto, não havendo qualquer tipo de intervenção nos procedimentos médicos ou de enfermagem, resultando em algumas perdas de informações por anotações incompletas, compensadas através de criteriosa revisão dos registros de dispensação e devolução de medicamentos à farmácia hospitalar.
Após análise estatística de dados, não houve diferença na porcentagem de pacientes com dor e sem dor no POI, acompanhados na REPAI, entre o grupo que recebeu dipirona e o que recebeu dipirona associada ao cetoprofeno, diante disso, conclui-se que não há diferença na eficácia analgésica do segundo grupo em relação ao primeiro, característica que se mantém nas etapas seguintes. No que diz respeito ao cetoprofeno, que por suas propriedades anti-inflamatórias deveria alterar os padrões de dor obtidos a partir do $1^{\circ} \mathrm{POI}$, não houve influência significativa da ação deste fármaco no número de pacientes com dor, em desacordo com os resultados de Zippel (2007), Moore (2008) e Rang (2012) $(11,22,23)$.

Da mesma forma, o tramadol não apresentou açāo mais efetiva que os demais fármacos utilizados. Quando administrado isoladamente ou associado à dipirona ou ainda ao cetoprofeno, o tramadol não apresentou eficácia superior, corroborando o relato de Thévenin e colaboradores (10), em ensaio publicado em 2008, no qual demonstrou que a DE80 (dose analgésica efetiva em $80 \%$ de uma população) é de $260 \mathrm{mg}$ por via endovenosa, dosagem muito acima da máxima recomendada pelos fabricantes, o equivalente à DE50, portanto, 100 mg e concluiu que não há indicação do uso de tramadol no tratamento da dor pós-operatória, pelo fato da dose eficaz (DE80) estar acima da recomendada e, consequentemente, apresentar maior risco de depressão respiratória entre outros efeitos colaterais no sistema nervoso central.

Em termos farmacológicos não é possível discorrer com clareza sobre qualquer tipo de interferência resultante da associação da dipirona com o cetoprofeno, por serem fármacos de famílias químicas e de mecanismos de ação diferentes. A dipirona é derivada das pirazolonas, enquanto o cetoprofeno vem da família do ácido propiônico. O mecanismo de ação da dipirona baseia-se na ativação de um complexo arginina/cGMP em receptor periférico com potencialização do efeito antinociceptivo em neurônio espinal, ao passo que, o cetoprofeno é um inibidor de ciclo-oxigenase 1 e 2 e lipoxigenases $(7,25,26)$. Quanto ao uso de associações, tais como, dipirona com tramadol ou dipirona com tramadol e cetoprofeno, não foi possível apontar interferência inerente ao modo de ação de qualquer um sobre os outros. O tramadol é um derivado da morfina, possui ação central sobre receptores opióides e não concorre pelos mesmos receptores ou sítios analgésicos dos outros fármacos estudados neste trabalho (23).

Em uma metanálise realizada em 2010, concluiu-se que existe informação insuficiente para uma análise segura devido ao reduzido número de pacientes nos estudos já realizados. Uma dose simples de Dipi- 
rona $500 \mathrm{mg}$, segundo a revisão sistemática, confere boa analgesia para grande porcentagem de pacientes, mas há necessidade de mais evidências sobre os reais benefícios do uso de altas doses de Dipirona e seu potencial prejuízo aos pacientes. Em vários países outras drogas com mais evidência científica de benefício estão disponíveis e provavelmente deveriam ser eleitas em detrimento da Dipirona. Optando-se pelo uso da Dipirona, pacientes devem ser monitorados para discrasias sanguíneas (8).

Neste trabalho, a porcentagem de pacientes avaliados que se queixaram de dor aguda contínua, após 48 horas do procedimento cirúrgico, ou seja, no $3^{\circ} \mathrm{PO}$ foi estatisticamente significativa, em discordância com a conclusão de Bierhals et al, em 1999 (4).

É de extrema importância ressaltar que embora o tempo de duração da dor aguda tenha se mostrado mais prolongado que o esperado, o número de pacientes apresentando dor pós-operatória neste estudo ficou na ordem de $30 \%$, resultado comparável ao menor índice encontrado na literatura internacional $(14,15,16,17,18)$.

\section{REFERÊNCIAS}

1. Lawrence K. Psiquiatria Clínica. 1th ed. Rio de Janeiro: Interamericana; 1981.

2. Pimenta CAM. Dor: Manual Clínico de Enfermagem. 1th ed. SN, editor. São Paulo; 2000.

3. Moreno JR. A Teoria Moderna da Dor e suas Consequências Práticas. Prática Hospitalar. 2004. 35:1-9.

4. Bierhals,N; Carvalho A; Willi J. Manejo da Dor Pós- operatória: Opióides x Cuidados de Enfermagem. Forum Enfermagem [Internet]. 1999 [cited 2011 Aug, 25]; Available from: http://www. forumenfermagem.org/index.php?option $=\mathrm{com}_{-}$ content\&task=view\&id=3202.

5. Carvalho J. Da farmácia, origem e evolução. 2nd ed. Rio de Janeiro: Tribuna Farmacêutica. 1924.

6. Solomon P; Patch VD. Manual de psiquiatria. 1a ed. Mexico DF: El Manual Moderno; 1972.

7. Budavavi $\mathrm{S}$ et all. The merck Index. An Encycolpedia of Chemicals Drugs, And Biologicals. 12a ed. New Jersey: Merck Research Laboratories; 1996.

\section{CONCLUSÃO}

O estudo demonstrou que a incidência de dor aguda contínua pós-operatória nos pacientes das Unidades de Internação de Cirurgia do Aparelho Digestivo e Cirurgia Geral do Hospital de Clínicas da Universidade Federal do Paraná tratados com os analgésicos e anti-inflamatórios padronizados neste hospital foi de trinta por cento, dentro da margem indicada na literatura internacional e portanto, o protocolo farmacoterapêutico é eficaz.

\section{AGRADECIMENTOS}

Prof $^{\mathrm{a}} \operatorname{Dr}^{\mathrm{a}}$ Heda Maria Barska dos Santos Amarante, Diretora Geral do HC; Prof. Dr. Antonio Carlos L. Campos, Prof. Dr. Jorge Eduardo F. Matias, Programa de Pós-graduação em Clínica Cirúrgica; servidores e funcionários do HC da UFPR.

Centro de Informação sobre Medicamentos do Conselho Regional de Farmácia do Estado do Paraná (CIM-CRF- PR).

Cláudia Maria Schiavon Bezerra pelo auxílio na análise estatística.
8. Edwards J; Meseguer F; Faura C; Moore RA; McQuay HJ; Derry S. Single dose dipyrone for acute postoperative pain. Cochrane database of systematic reviews (Online) [Internet]. 2010 Jan [cited 2011 Aug 25];(9):CD003227. Available from: http:// www.ncbi. nlm.nih.gov/pubmed/20824835.

9. Fuchs FD; Wannamacher L. Farmacologia Clínica. 4a ed. Rio de Janeiro: Guanabara-Koogan; 2010.

10. Thévenin A; Beloeil H; Blanie A; Benhamou D; Mazoit J-X. The limited efficacy of tramadol in postoperative patients: a study of ED80 using the continual reassessment method. Anesthesia and analgesia. 2008. 106(2):622-627.

11. Moore RA; Edwards JE; Meseguer F; Faura CC; McQuay HJ. Single-dose dipyrone for acute postoperative pain. Cochrane database of systematic reviews (Online) [Internet]. 2001 Jan [cited 2012 Aug 6];(3):CD003227. Available from: http://www.ncbi. nlm.nih.gov/pubmed/11687057. 
12. Bagán JV; Arranz JSL; Valencia E; Santamaría J; Eguidazu I; Horas M; et al. Clinical comparison of dexketoprofen trometamol and dipyrone in postoperative dental pain. J Clin Pharmacol. 1998. 38(12 Suppl):55S-64S.

13. Drugdex System. Micromedex, Thomson [Internet]. 2012 [cited 2013 Mar 13]. Available from: http:// www.thomsonhc.com.

14. Juhl IU; Christensen BV; Bülow HH; Wilbek H; Dreijer NC; Egelund B. Postoperative pain relief, from the patients' and the nurses' point of view. Acta Anaesthesiol Scand. 1993. 37(4):404-409.

15. Joshi GP. Postoperative pain management. Int Anesthesiol Clinics. 1994. 32(3):113-126.

16. Sjotröm B; Haljamae H; Dahlgren L-O; Lindström B. Assessment of postoperative pain: impact of clinical experience and professional role. Acta Anaesthesiol Scand. 1997. 41(3):339-344.

17. Dolin SJ; Cashman JN; Bland JM. Effectiveness of acute postoperative pain management: I. Evidence from published data. Brit J Anaesthesia. 2002. 89(3):409-423.

18. Power I. Recent advances in postoperative pain therapy. Brit J Anaesthesia. 2005. 95(1):43-51.

19. Moraes VC Bassi DU; Brandão DF; Secoli S. Perfil da terapia analgésica utilizada na dor pós-operatória de hemorroidectomia. Ciência, Cuidado e Saúde. 2008. 7(4):454-460.
20. Secoli SE all. Dor pós-operatória: combinações analgésicas e eventos adversos. Rev Escola de Enfermagem USP. 2009. 43:1244-1249.

21. Zylber-Katz E; Caraco Y; Granit L; Levy M. Dipyrone metabolism in liver disease. Clin Pharmacol Therapeutics. 1995. 58(2):198-209.

22. Zippel H; Wagenitz A. Comparison of the efficacy and safety of intravenously administered dexketoprofen trometamol and ketoprofen in the management of pain after orthopaedic surgery: A multicentre, double-blind, randomised, parallel-group clinical trial. Clin Drug Investig. 2006. 26(9):517528.

23. Rang HP; Dale MM; Ritter JM. Farmacologia. $6^{\text {a }}$ ed. São Paulo: Elsevier; 2007.

24. Shir Y; Rosen G; Zeldin A; Davidson EM. Methadone is safe for treating hospitalized patients with severe pain. Can J Anaesthesia. 2001. 48(11):11091113.

25. Katzung B. Basic and Clinical Pharmacology. 11th ed. San Francisco, CA: Mc Graw Hill; 2009.

26. Lorenzetti BB; Ferreira SH. Activation of the arginine- nitric oxide pathway in primary sensory neurons contributes to dipyrone-induced spinal and peripheral analgesia. Inflammation Res. 1996. 45(6):308311. 